\title{
Face masks in radiation oncology clinics: based on evidence or source of mistakes?
}

\author{
Rainer J. Klement ${ }^{1}{ }^{\mathbb{D}} \cdot$ Reinhart A. Sweeney $^{1}$
}

Received: 2 June 2020 / Accepted: 23 July 2020 / Published online: 1 August 2020

(c) Springer Science+Business Media, LLC, part of Springer Nature 2020

\begin{abstract}
We here express our concern about a general decree to let patients wear face masks in radiation oncology clinics. We believe that potential risks associated with wearing masks, such as the risk of confounding patients, outweigh any benefits of such a policy for which evidence of protection from COVID-19 is generally weak. For asymptomatic patients, wearing masks in addition to hygiene standards will not provide additional protection of others and should be cautioned against.
\end{abstract}

Keywords COVID-19 $\cdot$ Face masks $\cdot$ Radiation oncology $\cdot$ SARS-CoV-2

To the editor,

With some concern, we have read recent recommendations for altering radiation treatment techniques and procedures during the coronavirus disease 2019 (COVID-19) crisis. These include "avoiding use of gated breath hold techniques which require extensive therapist's effort" or "optimal use of faster orthogonal KV imaging in preference to daily CBCT" [1]. These measures are proposed in order to avoid treatment errors that may be caused by staff members due to a general tendency to minimize patient contact and protective clothes such as face masks, leading to fogged eyeglasses, inconvenience of wearing them, and decreased focus of attention [1]. A related problem arises from the fact that patients wearing masks are prone to being confused with another patient, increasing the potential for delivering the wrong treatment plan and causing great harm.

We here question the general requirement for patients and staff having to wear masks without being symptomatic. The reason is that we could not find solid evidence for the efficacy of masks against respiratory virus infection. Meta-analyses and systematic reviews of randomized controlled trials could not establish a protective effect of medical masks or N95 respirators against influenza or other respiratory virus infections [2,3]. While observational studies related to the

Rainer J. Klement

rainer_klement@gmx.de

1 Department of Radiotherapy and Radiation Oncology, Leopoldina Hospital Schweinfurt, Robert-Koch-Straße 10, 97422 Schweinfurt, Germany
2003 SARS-CoV-1 indicated an overall protective effect of medical masks, these studies were prone to bias; furthermore, no protection against SARS-CoV-1 was found for disposable, cotton, or paper masks [3]. Leung et al. [4] recently showed that mask wearing significantly reduces the viral load of coronaviruses in aerosols $(\leq 5 \mu \mathrm{m})(\mathrm{p}=0.02)$, but not respiratory droplets $(>5 \mu \mathrm{m})(\mathrm{p}=0.07)$ in infected symptomatic individuals. However, viral load measured over $30 \mathrm{~min}$ was generally small, and 8 of $23(35 \%)$ individuals with coronavirus did not shed any detectable virus in respiratory material at all; the same was true for four individuals with coronavirus who did not cough during the experiment. Thus, translated to COVID-19, face masks may protect others from infection if the wearer has clear symptoms of respiratory disease, but evidence for self-protection through masks in addition to hygiene standards such as hand washing and distance keeping is absent or weak, in particular when masks do not fulfill medical standards or are used accordingly. Mechanistically, with 60-140 nm [5], the SARS-CoV-2 virus would be able to penetrate even medical masks to a significant degree [6]. We, therefore, think that the potential danger of confusing patients outweighs the putative benefits of wearing a mask for asymptomatic patients. Although there exist some forms of (semi-)automatic patient identification tools which tie treatment parameters to, for example, anatomic specifics (iris, palmar venous pattern, etc.) or some nonanatomic pattern (bar code), few departments use these routinely and even these can only be part of a complete solution. Also, face masks may cover otherwise obvious pathologic 
clinical symptoms such as anemia, weight loss/Cushing's symptomatic etc.

Thus, even in critical times, evidence-based medicine, radiation safety, and our quite subtle and sensitive medical awareness should not be forgotten. We strongly caution against a general decree of mask wearing in radiation oncology clinics.

Author contributions Both authors wrote and approved the manuscript.

Funding No funding was received for writing this article.

Data availability Not applicable.

Code availability Not applicable.

\section{Compliance with ethical standards}

Conflict of interest The authors declare that they have no conflicts of interest.

\section{References}

1. Mohindra P, Buckey CR, Chen S, Sio TN, Rong Y. Radiation therapy considerations during the COVID-19 Pandemic: literature review and expert opinions. J Appl Clin Med Phys. 2020;21(5):612. https://doi.org/10.1002/acm2.12898.

2. Bin-Reza F, Lopez Chavarrias V, Nicoll A, Chamberland ME. The use of masks and respirators to prevent transmission of influenza: a systematic review of the scientific evidence. Influenza Other Respi Viruses. 2012;6:257-67. https://doi.org/10.111 1/j.1750-2659.2011.00307.x.

3. Offeddu V, Yung CF, Low MSF, Tam CC. Effectiveness of masks and respirators against respiratory infections in healthcare workers: a systematic review and meta-analysis. Clin Infect Dis. 2017;65:1934-42. https://doi.org/10.1093/cid/cix681.

4. Leung NHL, Chu DKW, Shiu EYC, Chan K, McDevitt JJ, Hau $\mathrm{BJP}$, et al. Respiratory virus shedding in exhaled breath and efficacy of face masks. Nat Med. 2020;26:676-80. https://doi. org/10.1038/s41591-020-0843-2.

5. Zhu N, Zhang D, Wang W, Li X, Yang B, Song J, et al. A novel coronavirus from patients with pneumonia in China, 2019. N Engl J Med. 2020;382:727-33. https://doi.org/10.1056/NEJMoa2001 017.

6. Bałazy A, Toivola M, Adhikari A, Sivasubramani SK, Reponen T, Grinshpun SA. Do N95 respirators provide 95\% protection level against airborne viruses, and how adequate are surgical masks? Am J Infect Control. 2006;34:51-7. https://doi.org/10.1016/j. ajic.2005.08.018

Publisher's Note Springer Nature remains neutral with regard to jurisdictional claims in published maps and institutional affiliations. 